\title{
Is diversity harmful? - Mixed-brand cardiac implantable electronic devices undergoing magnetic resonance imaging
}

\author{
Christoph Alexander König · Florian Tinhofer · Thomas Puntus · Achim Leo Burger · Nikolaus Neubauer · \\ Herbert Langenberger · Kurt Huber · Michael Nürnberg · David Zweiker (D)
}

Received: 22 March 2021 / Accepted: 9 July 2021 / Published online: 17 August 2021

(C) The Author(s) 2021

\begin{abstract}
Summary
Background Many patients with cardiac implantable electronic devices (CIED) undergo magnetic resonance imaging (MRI); however, a relevant proportion have a CIED system that has not been classified as MRI-conditional because of generators and leads from different brands (mixed-brand group). The available data concerning the outcome of these mixed patients undergoing MRI is limited.

Methods A retrospective single center study, including all patients with CIEDs undergoing MRI between January 2013 until May 2020, was performed. Primary endpoints were defined as death or any adverse event necessitating hospitalization or CIED revision. Secondary endpoints were the occurrence of any sign for

The data were collected as part of the diploma thesis of Christoph A. König.
\end{abstract}

\section{Supplementary Information The online version of this article (https://doi.org/10.1007/s00508-021-01924-w) contains supplementary material, which is available to authorized users.}

C. A. König · F. Tinhofer · T. Puntus · A. L. Burger · K. Huber · M. Nürnberg · Dr. med.univ. D. Zweiker $(\square)$ 3rd Department of Medicine, Cardiology and Intensive Care Medicine, Klinik Ottakring (Wilhelminenhospital),

Montleartstraße 37, 1160 Vienna, Austria

david.zweiker@medunigraz.at

C. A. König

Medical University of Vienna, Vienna, Austria

N. Neubauer $\cdot$ H. Langenberger

Institute for Diagnostic and Interventional Radiology, Klinik Ottakring (Wilhelminenhospital), Vienna, Austria

K. Huber

Medical School, Sigmund Freud University, Vienna, Austria

Dr. med.univ. D. Zweiker

Division of Cardiology, Medical University of Graz, Graz, Austria beginning device or lead failure or patient discomfort during MRI.

Results A total of 227 MRI examinations, including 10 thoracic MRIs, were carried out in 158 patients, with 1-9 MRIs per patient. Of the patients 38 underwent 54 procedures in the mixed-brand group and 89 patients underwent 134 MRIs in the MRI-conditional group. Of the patients 31 were excluded since the MRI conditionality could not be determined. No primary endpoints occurred within the mixed-brand group but in $2.2 \%$ of the MRI-conditional group ( $p=$ 1.000), with 2 patients developing new atrial fibrillation during MRI, of whom one additionally had a transient CIED dysfunction. No secondary endpoints were met in the mixed-brand group compared to $3.4 \%$ in the MRI-conditional group $(p=0.554)$. No complications occurred in the excluded patients.

Conclusion The complication rate of CIED patients undergoing MRI was low. Patients with a mixed CIED system showed no signs of increased risk of adverse events compared to patients with MRI-conditional CIED systems.

Keywords Magnetic resonance imaging • Pacemaker · Defibrillator · MRI-conditional · Safety

\section{Introduction}

Magnetic resonance imaging (MRI) is an important instrument in radiology and increasingly used in daily clinical practice [1]. More than 30 million MRI scans are performed each year in the European Union [2]. Simultaneously, the number of patients with cardiac implantable electronic devices (CIED), such as pacemakers and defibrillators, is increasing quickly as well. Thus, it is estimated that $50-75 \%$ of the patients with an CIED need an MRI once in the lifetime of their device [3]. Previously, MRI was contraindicated in the 
presence of an CIED [4], as generator failure, newonset arrhythmia and even death were feared [5-7]. Due to advances in technology, manufacturers started with the development of so-called MRI-conditional CIED systems since 2010, minimizing the risks of undergoing MRI with such a CIED system in place, as long as certain specific conditions are fulfilled $[8,9]$. Many studies have already demonstrated that the risks of suffering any side effects when undergoing MRI with an MRI-conditional CIED system in place are negligible. [9-13]. Currently, most of the available CIED systems are considered MRI-conditional; however, by 2011 approximately 1.8 million CIED systems were in use that were not MRI-conditional in the USA alone [14]. Although formally unsuitable for undergoing MRI, the risks for suffering any adverse events with an MRI-unconditional CIED system remain very low, as several studies impressively proved [7, 15-17].

A MRI-conditional CIED system refers to both the generator and the attached leads, which are approved only in a certain combination and the absence of abandoned leads [5]; however, a significant proportion of patients have a combination of per se MRIconditional elements from different manufacturers (mixed-brand group) [18]. Current guidelines endorse performing MRI in these patients as off-label use, if the value of MRI outweighs the small risk of device failure or damage [19] but clinical data are scarce. Therefore, this monocentric analysis was performed to assess possible complications in patients with a mixed-brand CIED system undergoing MRI compared to patients with completely MRI-certified systems.

\section{Material and methods}

This retrospective cohort study included all patients with a CIED who underwent MRI from January 2013 until May 2020 at a major public hospital (Klinik Ottakring, Vienna, Austria). According to the responsible ethics committee, which approved the study (no. 20137-VK), no informed consent was necessary.

\section{Clinical workflow at the institution}

Patients with a CIED and the need to undergo an MRI were presented to the institution's department of cardiology. Based on the clinical indications for the MRI and the type of CIED implanted, a decision was made to accept or cancel the MRI procedure based on an individual risk-benefit analysis. The main criteria for acceptance were the absence of abandoned leads and the therapeutic consequence of the MRI examination. If in doubt, the radiologist and the physician responsible for the indications were contacted and the final decision was made following an interdisciplinary discussion. Before MRI, the CIED system was checked and put into MRI mode (if available) and either asynchronous pacing mode (V00 or D00) or the suspension of pacing therapy (000) was programmed. In patients with an implantable cardiac defibrillator (ICD), antitachycardia treatment was deactivated. and patients were continuously accompanied by trained health personnel. During MRI, the electrocardiogram and oxygen saturation were continuously monitored. Immediately after MRI, the CIED was checked and previous settings were restored. The next follow-up was planned after 3-12 months according to the type of CIED and clinical indications.

All MRI examinations were conducted with an MRI scanner with a magnetic field strength of $1.5 \mathrm{~T}$, a maximum gradient field strength of $45 \mathrm{mT} / \mathrm{m}$ and a maximum gradient slew rate of $200 \mathrm{~T} / \mathrm{m} / \mathrm{s}$ using solely receiving coils.

Usually, standard MRI protocols were used, following the current guidelines [5], ensuring a maximum whole body SAR (Specific Absorption Rate) of $<2 \mathrm{~W} / \mathrm{kg}$ and a head SAR of $<3.2 \mathrm{~W} / \mathrm{kg}$, as well as minimizing the number of sequences and the scanning duration.

\section{Patient groups}

Patients were stratified into three groups according to the MRI-conditionality of the patient's CIED system. All patients who had a completely MRI-conditional system according to the manufacturers' current recommendations were classified into the MRI-conditional group. Patients with MRI-conditional elements but different manufacturers were included into the mixed-brands group. Remaining patients with CIED elements without or with unclear MRI certification were excluded from the primary analysis, but adverse events were still documented (excluded group).

\section{Data collection}

All patients with CIED who received an MRI between 1 January 2013 and 31 May 2020 were included. For analysis of complications after MRI, the patient's health records within all Viennese public hospitals were reviewed for admissions for a period up to 1 year after the MRI scan.

\section{Endpoints}

As primary endpoint, the occurrence of any complication of a CIED after MRI requiring an intervention (e.g., device failure, lead failure, device dysfunction, or any other periprocedural or postprocedural complication, such as newly developed atrial fibrillation) was compared in MRI-conditional vs. mixed-brand groups.

Secondary endpoints were the occurrence of any sign for beginning device or lead failure or minor clinical conditions. Changes in lead performance were determined on the difference in parameters between pre-MRI and post-MRI as defined by the American Heart Rhythm Society Consensus Statement [5]. An 
original article

Table 1 Baseline characteristics of all included patients, mixed-brand and MRI-conditional groups

\begin{tabular}{|c|c|c|c|c|}
\hline Parameter & All included patients & Mixed-brand group & MRI-conditional group & $P$ value \\
\hline \multicolumn{5}{|l|}{ Demographics } \\
\hline Number of patients & 127 & 38 & 89 & $\mathrm{~N} / \mathrm{A}$ \\
\hline Number of MRI procedures & 188 & 54 & 134 & N/A \\
\hline Age (years) (interquartile range) & $73(67-80)$ & $77(73-81)$ & $72(64-79)$ & $0.003^{*}$ \\
\hline Female gender & $34.6 \%$ & $39.5 \%$ & $32.6 \%$ & 0.542 \\
\hline Height $(\mathrm{cm})^{\mathrm{a}}( \pm$ standard deviation) & $173 \pm 8$ & $173 \pm 6$ & $173 \pm 9$ & 0.935 \\
\hline Weight (kg) ${ }^{\mathrm{a}}$ (interquartile range) & $80(73-84)$ & $78(71-80)$ & $82(75-93)$ & 0.126 \\
\hline \multicolumn{5}{|l|}{ CIED details } \\
\hline CIED type $e^{\mathrm{a}}$ & & & & 0.338 \\
\hline Pacemaker & $92.1 \%$ & $97.4 \%$ & $89.9 \%$ & - \\
\hline Transvenous ICD & $6.3 \%$ & $2.6 \%$ & $7.9 \%$ & - \\
\hline Subcutaneous ICD & $1.6 \%$ & $0 \%$ & $2.2 \%$ & - \\
\hline \multicolumn{4}{|l|}{ Indication $^{\mathrm{a}}$} & $<0.001$ \\
\hline Sick sinus syndrome & $30.8 \%$ & $17.2 \%$ & $35.9 \%$ & - \\
\hline Atrioventricular block & $27.1 \%$ & $20.7 \%$ & $29.5 \%$ & - \\
\hline Bradycardia-tachycardia syndrome & $23.4 \%$ & $37.9 \%$ & $17.9 \%$ & - \\
\hline Other block & $4.7 \%$ & $13.8 \%$ & $1.3 \%$ & - \\
\hline Heart failure & $3.7 \%$ & $3.4 \%$ & $3.8 \%$ & - \\
\hline Secondary prophylaxis & $3.7 \%$ & $3.4 \%$ & $3.8 \%$ & - \\
\hline Other Indication & $6.5 \%$ & $3.4 \%$ & $7.7 \%$ & - \\
\hline \multicolumn{4}{|l|}{ Generator brand ${ }^{a}$} & $<0.001$ \\
\hline Biotronik $^{b}$ & $32.3 \%$ & $5.3 \%$ & $43.8 \%$ & - \\
\hline Boston Scientific ${ }^{\mathrm{C}}$ & $12.6 \%$ & $10.5 \%$ & $13.5 \%$ & - \\
\hline Medtronic $^{d}$ & $24.4 \%$ & $36.8 \%$ & $19.1 \%$ & - \\
\hline Sorin/LivaNova ${ }^{\mathrm{e}}$ & $17.3 \%$ & $42.1 \%$ & $6.7 \%$ & - \\
\hline St. Jude Medical/Abbott ${ }^{f}$ & $13.4 \%$ & $5.3 \%$ & $16.9 \%$ & - \\
\hline Months since prior box change before MRI & $26.8(12.1-48.1)$ & $33.0(11.4-48.9)$ & $25.4(12.3-47.9)$ & 0.953 \\
\hline Prior box change $<3$ months & $2.4 \%$ & $2.6 \%$ & $2.2 \%$ & 1.000 \\
\hline \multicolumn{4}{|l|}{ Lead brand ${ }^{a}$} & 0.002 \\
\hline Biotronik $^{b}$ & $54.3 \%$ & $78.9 \%$ & $43.8 \%$ & - \\
\hline Boston Scientific ${ }^{\mathrm{C}}$ & $10.2 \%$ & $2.6 \%$ & $13.5 \%$ & - \\
\hline Medtronic $^{d}$ & $18.1 \%$ & $15.8 \%$ & $19.1 \%$ & - \\
\hline Sorin/LivaNova ${ }^{\mathrm{e}}$ & $4.7 \%$ & $0.0 \%$ & $6.7 \%$ & - \\
\hline St. Jude Medical/Abbott ${ }^{f}$ & $12.6 \%$ & $2.6 \%$ & $16.9 \%$ & - \\
\hline Months since last lead implantation before MRI & $36.6(15.1-66.1)$ & $39.2(18.0-80.4)$ & $34.3(14.2-61.6)$ & 0.662 \\
\hline Last lead implantation $<3$ months & $0.8 \%$ & $2.6 \%$ & $0.0 \%$ & 0.299 \\
\hline \multicolumn{5}{|l|}{ MRI details } \\
\hline \multicolumn{4}{|l|}{ Location of MRI examination } & 0.322 \\
\hline Head or extremities & $40.4 \%$ & $48.1 \%$ & $37.3 \%$ & - \\
\hline Extrathoracic torso & $55.3 \%$ & $50.0 \%$ & $57.5 \%$ & - \\
\hline Thorax & $4.3 \%$ & $1.9 \%$ & $5.2 \%$ & - \\
\hline \multicolumn{4}{|l|}{ Pacing mode during $M R^{\circledR}$} & 0.681 \\
\hline 000 & $7.4 \%$ & $10.0 \%$ & $6.8 \%$ & - \\
\hline A00/N00 & $15.7 \%$ & $10.0 \%$ & $17.0 \%$ & - \\
\hline D00 & $76.9 \%$ & $80.0 \%$ & $76.1 \%$ & - \\
\hline Estimated ERI before MRI (years) & $7.4(6.0-9.3)$ & $7.0(5.0-10.3)$ & $7.5(6.0-9.1)$ & 0.831 \\
\hline \multicolumn{5}{|c|}{ 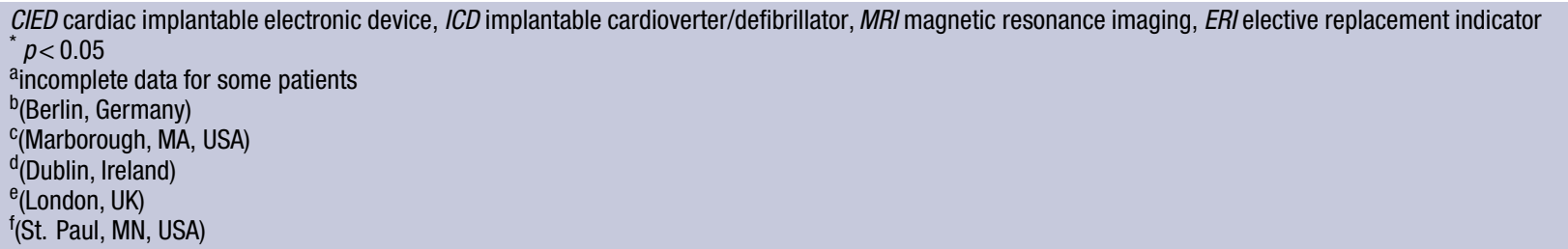 } \\
\hline
\end{tabular}


Table 2 Outcome of included patients, mixed-brands and MRI-conditional groups

\begin{tabular}{|c|c|c|c|c|}
\hline Parameter & All included patients (\%) & Mixed-brands group (\%) & MRI-conditional group (\%) & $P$ value \\
\hline \multicolumn{5}{|l|}{ Shot-term outcome } \\
\hline Primary endpoints & 1.6 & 0.0 & 2.2 & 1.000 \\
\hline Secondary endpoints & 2.4 & 0.0 & 3.4 & 0.554 \\
\hline Patient discomfort & 1.6 & 0.0 & 2.2 & 1.000 \\
\hline Increase of ventricular threshold & 0.8 & 0.0 & 1.1 & 1.000 \\
\hline \multicolumn{5}{|l|}{ Long-term follow-up } \\
\hline Decrease of amplitude > 50\% & 4.7 & 7.9 & 3.4 & 0.363 \\
\hline Increase of pacing threshold $>1 \mathrm{~V}$ & 3.1 & 0.0 & 4.5 & 0.316 \\
\hline 1-year rehospitalization & 37.8 & 36.8 & 38.2 & 1.000 \\
\hline
\end{tabular}

increase in the pacing lead threshold by $1.0 \mathrm{~V}$, a decrease in the $\mathrm{P}$-wave or R-wave amplitude by $50 \%$, a pacing lead impedance change by $\pm 50 \Omega$ or a highvoltage (shock) lead impedance change by $\pm 5 \Omega$ were considered relevant.

\section{Statistical analysis}

Results were given as mean (standard deviation), median (interquartile range) or proportion, where appropriate. Bivariate analysis of baseline characteristics and outcome between MRI-conditional vs. mixedbrand groups was performed using parametric and non-parametric tests, where appropriate. Fisher's exact test was used to compare differences of the occurrence of the primary and secondary outcome between both groups. Data were analyzed based on each patient, but the MRI details section of Tables 1 and 2 were calculated based on each MRI examination. All statistical analyses were performed with R 4.0.3 (The R Foundation, Vienna, Austria).

\section{Results}

In the study period, a total of $227 \mathrm{MRI}$ examinations were performed in 158 different CIED patients, with a range of 1-9 MRI procedures per patient. The total yearly number of MRI examinations rose from 15 in 2013 to 64 in 2018. Of all procedures, 134 MRI procedures in 89 patients were stratified into the MRIconditional group, while $54 \mathrm{MRI}$ procedures in 38 patients were included in the mixed-brand group. The MRI-conditionality could not be determined in 31 patients undergoing $39 \mathrm{MRI}$ procedures and were therefore excluded from the primary analysis. In the MRIconditional group, 7 patients $(5.2 \%)$ underwent a thoracic MRI, including 5 specifically cardiac MRIs. In the mixed-brand group, there was one patient (1.9\%) who received a cardiac MRI. There were no MRIs found that were performed on patients with abandoned leads present. A study flowchart is presented in Fig. 1.

\section{Demographics}

The overall mean age was 73 years, with patients in the mixed-brand group being significantly older than patients of the conditional group (77 years vs. 72 years, $p=0.003$ ). Mean height was $173 \mathrm{~cm}$, median weight was $80 \mathrm{~kg}$ and $34.6 \%$ of patients were female, with no significant differences between groups (Table 1). Baseline characteristics of excluded patients are available in Supplemental Table 1.

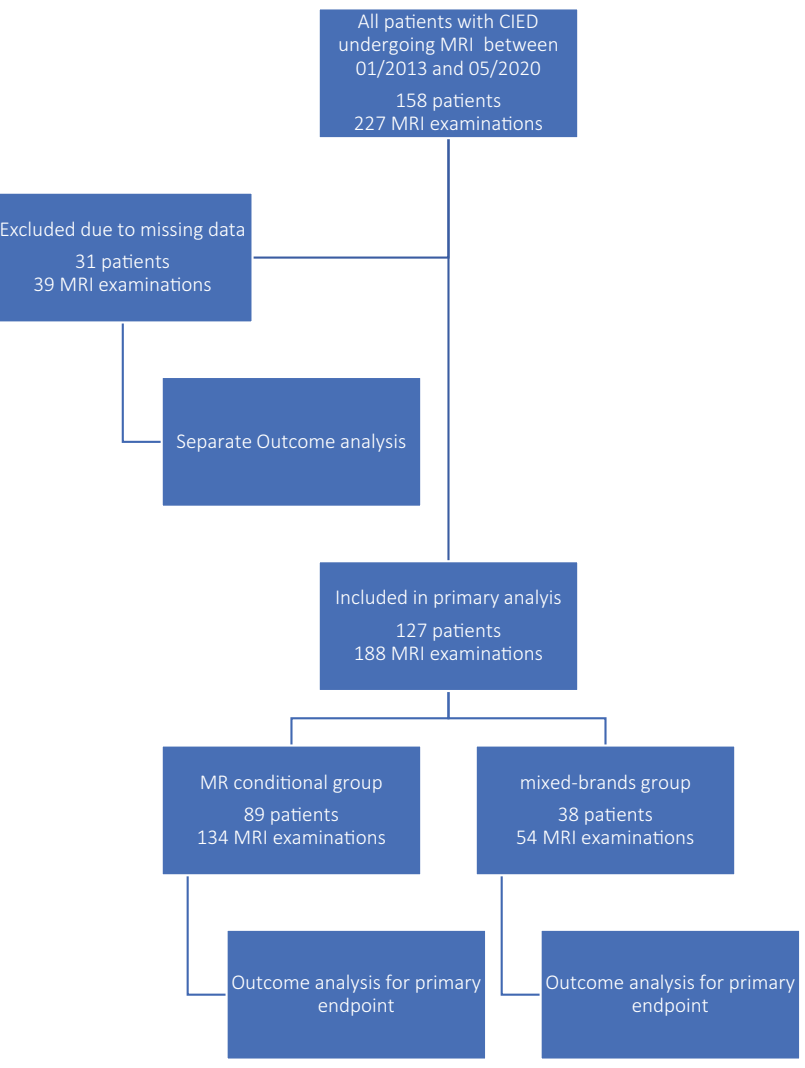

Fig. 1 Study flowchart. CIED cardiac implantable electric device, $M R I$ magnetic resonance imaging 


\section{CIED details}

Within all the included patients, $92.1 \%$ of the CIEDs were pacemakers and $7.9 \%$ were ICDs. The most common indications for CIED implantation within the included patients were sick sinus syndrome (30.8\%), followed by atrioventricular block $(27.1 \%)$ and bradycardia-tachycardia syndrome (23.4\%) (Table 1). In total, within all the included patients, the most common pacemaker brand, concerning both generators $(29.9 \%)$ and leads $(54.7 \%)$, was Biotronik (Berlin, Germany). Notable are the significant differences between the different groups, with $42.5 \%$ of the pacemaker generators from Biotronik in the MRIconditional group, compared to only $2.7 \%$ pacemaker generators from Biotronik in the mixed-brands group, where most generators (43.2\%) were from Sorin/Liva Nova (London, United Kingdom) instead. The lead brands too showed significant differences between the different groups, with $42.5 \%$ pacemaker leads from Biotronik in the MRI-conditional group, compared to $81.1 \%$ pacemaker leads, also from Biotronik, in the mixed-brands group. Additional details concerning generator and lead distribution are found in Supplemental Table 2 .

For ICDs, solely generators from Biotronik $(60.0 \%)$ and Boston Scientific (Natick, MA, USA) (40.0\%) were used, with leads mostly from Biotronik (50.0\%), followed by Boston Scientific (40.0\%). Here too, additional CIED details, stratified by pacemakers and ICDs, as well as groups, are given in Supplemental Table 2.

The time between the last generator or lead implantation and the MRI was on average 32.5 months and 45.3 months, with $1.6 \%$ and $0.5 \%$, respectively, having received the last procedure within the last 6 weeks. The mean time of the elective replacement indicator (ERI) was 7.5 years.

\section{Short-term outcome}

Primary endpoints were met in two patients (1.6\%), with no endpoints in the mixed-brand group $(0.0 \%)$ and two in the MRI-conditional group $(2.2 \%, p=1.000$, Table 2). One patient with a dual-chamber CRT-D, receiving a cranial MRI, developed de novo atrial fibrillation with fast ventricular conduction during the MRI examination. During the postprocedural CIED examination, the impedance of the right ventricular defibrillation electrode was not measurable and a marked drop in battery capacity above the elective replacement indicated (ERI) threshold was noted. The patient was hospitalized for one night until spontaneous conversion into sinus rhythm. The treating clinicians suspected the MRI pacing mode (D00 instead of 000 despite spontaneous sinus rhythm) to have caused the arrhythmia; however, the CIED examination before discharge showed normal measurements regarding the battery and electrodes. Since the atrial fibrilla- tion was considered to be triggered by CIED dysfunction, no anticoagulation treatment was established. At follow-up no further atrial arrhythmias were documented.

In another case, the device check following an unremarkable knee MRI examination revealed de novo atrial fibrillation with activation of mode switch. Anticoagulation treatment had already been established previously due to an indication other than atrial fibrillation and the follow-up after 1 year showed no further atrial arrhythmias.

In the short-term outcome, secondary endpoints occurred in three patients during three MRI examinations $(2.4 \%)$, also solely in the MRI-conditional group $(p=0.554)$. One patient with a lumbar MRI developed stenocardia when switching the CIED into the MRI mode. In another patient, also during a lumbar MRI, the MRI had to be aborted because the patient felt heat in the generator area during the examination. Both patients had an unremarkable follow-up. No other patients showed any symptoms when undergoing MRI. In a third patient, an increase of the ventricular threshold was noted during a routine control 6 months after the MRI examination of the shoulder.

\section{Long-term outcome and changes in lead performance}

The long-term follow-up further revealed four additional cases of threshold increase $(3.1 \%)$ during the follow-up, several months after the MRI examination. All events occurred in patients with MRI-conditional CIEDs $(4.5 \%)$ compared to none in the mixed-brands group ( $0 \%, p=0.316$ ) Additionally, 6 more cases $(4.7 \%)$ of sensing amplitude decrease by $>50 \%$ were identified, 3 in the MRI-conditional and 3 in the mixedbrands group (3.4\% in MRI-conditional group vs. $7.9 \%$ in mixed-brands group, $p=0.363$ ). More details concerning the lead performance are given in Supplemental Table 3.

In a period of 1 year after MRI, 38\% of patients were hospitalized, without significant differences between groups (38.2\% vs. $36.8 \%, p=1.000)$.

\section{Events in excluded patients}

In the patient group where the MRI-conditionality could not be determined, neither primary nor secondary endpoints were met, nor were any changes in lead performance noticed.

\section{Discussion}

In this retrospective monocentric study, the safety of MRI-unconditional CIED systems due to mixedbrand components was compared with MRI-conditional CIED systems in daily clinical practice. The study included consecutive patients with a CIED un- 
dergoing MRI at our institution and found no sign of increased adverse events in the mixed-brand group.

\section{$M R I$ and CIEDs}

The possible interactions between the MRI unit and implantable electronic devices are diverse and must be considered carefully by treating physicians, as the possible risks a CIED patient is facing when undergoing MRI are many [20-22], which is why a short summary is provided here.

There are three basic physical forces that can cause electromagnetic interferences (EMI) of the CIED, namely the static magnetic field, time varying gradient magnetic fields and radiofrequency energies. The static magnetic field affects the ferromagnetic parts of the CIED and may lead to distorting or loosening of the implanted parts [23] or may affect the so-called reed switch [24] that can still be found in older CIEDs. Gradient magnetic fields on the other hand may induce currents in conductive wires and thus may lead to unwanted myocardial stimulation and induce arrhythmias [25]. The radiofrequency energy, however, may lead to excessive heating of the local tissue [26], possibly even leading to tissue damage, especially when causing a so-called antenna effect [27]. Other risks to keep in mind are battery depletion [28] or power-on reset (POR) [29] as well as imaging artefacts that may occur when performing MRI in regions with a CIED in situ, such as thoracic or cardiac MRI [30].

While the benefits of unitary CIED systems from one manufacturer are obvious, the combination of different brands often cannot be avoided. Reasons may range from unavailability of elements from specific brands up to the preference of the implanting physician. As current CIEDs have to conform with specific requirements [31] ensuring compatibility, patients with mixed-brand systems normally are not facing problems; however, in the era of MRI-conditional CIED devices, new challenges arise for both the patients and the treating physicians. While the decision to perform the MRI may be backed up by guidelines $[5,19]$, the use of CIED devices as off-label and the legal consequences should be kept in mind. This study shows that a high proportion $(30.2 \%)$ of patients are now affected by this problem.

There are only a handful of comparative studies, focusing on the different outcomes between MRI conditional vs. MRI unconditional CIEDs. Notably, there is Shah et al. from 2017, including 105 patients but without a follow-up investigation [32], then there is Han et al. in 2019 with a total of 35 patients and a follow-up of 1 month after MRI [33] and last there is Seewöster et al. from 2019, comparing a total of 200 consecutive patients, with a follow-up interrogation 6 months after the MRI examination [34]. None of these studies showed any significant differences between groups nor any significant adverse effects in general when undergoing MRI [32-34].
Those studies did not take into consideration that CIED systems using elements from different manufacturers (although the separate parts themselves are per se MRI-conditional) are formally not considered as MR-conditional as a whole [5] but should rather be considered as MRI-unconditional and thus the performing of MRI in these patients remains an off-label use.

To the authors knowledge, this is the first study to investigate the effect of mixed brand CIED systems undergoing MRI, incorporating detailed information on device and lead measurements; however, although the presence of mixed-brand systems represents novelty, the results are not surprising as the single components themselves are all MRI-conditional and there are several studies that already proved the relative safety of undergoing magnetic resonance imaging even with an MRI-unconditional CIED [7, 15-17]. Additionally, only standard MRI protocols were used, which is a further reason no adverse events were to be expected, as perhaps would have been the case when performing MRIs with a higher energy deposition or closer to the generator.

Fortunately, the results suggest that undergoing MRI was safe even in these mixed-brands group, with two primary endpoints met solely in the MRI-conditional group. Both times the patients developed de novo atrial fibrillation. Both patients had a CIED system implanted that was MRI-conditional with generators and leads from the same manufacturer. The rate of secondary endpoints was also low. No device or lead replacement was necessary after MRI. These results, demonstrating the relative safety of undergoing MRI with a CIED, coincide with other studies already conducted, both for MRI-conditional [9-13] and unconditional CIEDs [7, 15-17].

In general, there were no significant differences noted between pre-MRI and post-MRI parameters or between the MR-conditional and the mixed-brands group, concerning capture threshold, sensing or impedance. Most changes were in a clinically acceptable range and coincided with other large studies in terms of the type of observed events [7, 9-13, 15-17].

The study included a (albeit small) number of thoracic and cardiac MRIs. No endpoints were met in these patients, neither in the MRI-conditional nor the mixed-brand group. Even though the significance of these results is small, due to the small number of cardiac MRIs observed, the results agree with other studies with bigger sample sizes that also showed that cardiac magnetic resonance is possible on patients with CIEDs [34-36]. Whether any imaging artifacts occurred with these thoracic MRIs or not, was not assessed by this study.

In addition, this study included many patients with repeated MRI examinations and the effect of multiple MRIs on the CIED systems could be examined. Undergoing multiple MRI examinations did not seem 
to have any influence on the outcome, since the few observed endpoints occurred both with patients who received multiple MRIs as well as with patients who received only one single MRI examination. This is corresponding with previous studies that also included multiple MRI examinations [13, 16, 37] and showed that patients who underwent multiple MRIs (the maximum number in a single patient was 9 MRIs) did not suffer any adverse effects; however, the study cannot add much evidence on the effect of multiple MRI examinations in patients with mixed-brand ICDs, as there was only one patient with a mixed-brand ICD who underwent two MRI examinations without adverse effects.

\section{Limitations}

The main limitation is the retrospective nature of this study. Due to missing data, only 134 MRIs could be allocated to the MRI-conditional group and 54 MRIs into the mixed-brands group. 39 MRIs that had to be excluded from this study, since their MRI-conditionality could not be determined due to missing data. Fortunately, in uncategorized patients none of the discussed endpoints occurred.

Another limitation is certainly the imbalance between pacemakers and ICDs. With only one single ICD patient in the mixed-brand group, these results may not be transferred to other populations with a higher proportion of ICD patients.

Additionally, we faced difficulties collecting all the individual lead parameters during the follow-ups, due to ofttimes missing data; however, in the data available, no significant abnormalities were found.

\section{Conclusion}

Our analysis did not find any evidence of increased risk of adverse events in patients with mixed-brand CIED systems undergoing MRI compared to patients with MRI-conditional CIED systems. Overall, it can be concluded that when a CIED patient is properly examined according to the established guidelines, undergoing MRI is relatively safe, regardless of whether the patient undergoes MRI with an MRI-conditional or a formally unconditional mixed CIED system, using MRI-conditional components from different manufacturers.

After all, this study shows that diversity appears not to be harmful at all.

Funding Open access funding provided by Medical University of Graz.

Conflict of interest C.A. König, A.L. Burger, N. Neubauer, H. Langenberger and K. Huber declare that they have no competing interests. D. Zweiker has received speaker fees and/or travel grants from Daiichi Sankyo and research grants from Boston Scientific. F. Tinhofer has received speaker fees from Medtronic. T. Puntus has received speaker fees from
Biotronik. M. Nürnberg has received speaker fees from Abbott Laboratories, Microport, Biotronik, Medtronic and Boston Scientific.

Open Access This article is licensed under a Creative Commons Attribution 4.0 International License, which permits use, sharing, adaptation, distribution and reproduction in any medium or format, as long as you give appropriate credit to the original author(s) and the source, provide a link to the Creative Commons licence, and indicate if changes were made. The images or other third party material in this article are included in the article's Creative Commons licence, unless indicated otherwise in a credit line to the material. If material is not included in the article's Creative Commons licence and your intended use is not permitted by statutory regulation or exceeds the permitted use, you will need to obtain permission directly from the copyright holder. To view a copy of this licence, visit http://creativecommons.org/licenses/by/4.0/.

\section{References}

1. Sammet S. Magnetic resonance safety. Abdom Radiol (NY). 2016;41(3):444-51.

2. Eurostat. Medical technologies - examinations by medical imaging techniques (CT, MRI and PET). 2021. https:// ec.europa.eu/eurostat/databrowser/view/hlth_co_exam/ default/table?lang=en. Accessed 12 Mai2021.

3. Kalin R, Stanton MS. Current clinical issues for MRI scanning of pacemaker and defibrillator patients. Pacing Clin Electrophysiol. 2005;28(4):326-8.

4. Faris OP, Shein M. Food and Drug Administration perspective: magnetic resonance imaging of pacemaker and implantable cardioverter-defibrillator patients. Circulation. 2006;114(12):1232-3.

5. IndikJH, Gimbel JR, Abe H, Alkmim-Teixeira R, Birgersdotter-Green U, Clarke GD, et al. 2017 HRS expert consensus statement on magnetic resonance imaging and radiation exposure in patients with cardiovascular implantable electronic devices. Heart Rhythm. 2017;14(7):e97-e153.

6 . Russo RJ. Determining the risks of clinically indicated nonthoracic magnetic resonance imaging at $1.5 \mathrm{~T}$ for patients with pacemakers and implantable cardioverter-defibrillators: rationale and design of the MagnaSafe registry. Am Heart J.2013;165(3):266-72.

7. Russo RJ, Costa HS, Silva PD, Anderson JL, Arshad A, Biederman RW, et al. Assessing the risks associated with MRI in patients with a pacemaker or defibrillator. $\mathrm{N} \mathrm{Engl} \mathrm{J}$ Med. 2017;376(8):755-64.

8. Shinbane JS, Colletti PM, Shellock FG. Magnetic resonance imaging in patients with cardiac pacemakers: era of "MR Conditional" designs. J Cardiovasc Magn Reson. 2011;13:63.

9. WilkoffBL, Bello D, TaborskyM, Vymazal J, KanalE,HeuerH, et al. Magnetic resonance imaging in patients with a pacemaker system designed for the magnetic resonance environment. Heart Rhythm. 2011;8(1):65-73.

10. Bauer WR, Lau DH, Wollmann C, McGavigan A, Mansourati J, Reiter T, et al. Clinical safety of ProMRI implantable cardioverter-defibrillator systems during head and lower lumbar magnetic resonance imaging at 1.5 Tesla. SciRep. 2019;9(1):18243.

11. Nazarian S, Cantillon DJ, Woodard PK, Mela T, Cline AM, Strickberger AS. MRI safety for patients implanted with the MRI ready ICD system: MRI ready study results. JACC Clin Electrophysiol. 2019;5(8):935-43.

12. van Dijk VF, Delnoy P, Smit JJJ, Ramdat Misier RA, Elvan A, van Es HW, etal. Preliminary findings on the safety of 1.5 and 
3 Tesla magnetic resonance imaging in cardiac pacemaker patients. JCardiovasc Electrophysiol. 2017;28(7):806-10.

13. Williamson BD, Gohn DC, Ramza BM, Singh B, Zhong Y, Li S, et al. Real-world evaluation of magnetic resonance imaging in patients with a magnetic resonance imaging conditional pacemaker system: results of 4-year prospective follow-up in 2,629 patients. JACC Clin Electrophysiol. 2017;3(11):1231-9.

14. Nazarian S, Hansford R, Roguin A, Goldsher D, Zviman MM, Lardo AC, et al. A prospective evaluation of a protocol for magnetic resonance imaging of patients with implanted cardiac devices. Ann Intern Med. 2011;155(7):415-24.

15. Munawar DA, Chan JEZ, Emami M, Kadhim K, Khokhar K, O'Shea C, et al. Magnetic resonance imaging in non-conditional pacemakers and implantable cardioverter-defibrillators: a systematic review and meta-analysis. Europace. 2020;22(2):288-98.

16. Nazarian S, Hansford R, Rahsepar AA, Weltin V, McVeigh D, Gucuk Ipek E, et al. Safety of magnetic resonance imaging in patients with cardiac devices. $\mathrm{N}$ Engl J Med. 2017;377(26):2555-64.

17. Shah AD, Morris MA, Hirsh DS, Warnock M, Huang Y, Mollerus M, et al. Magnetic resonance imaging safety in nonconditional pacemaker and defibrillator recipients: a meta-analysis and systematic review. Heart Rhythm. 2018;15(7):1001-8.

18. Levine GN, Gomes AS, Arai AE, Bluemke DA, Flamm SD, Kanal E, et al. Safety of magnetic resonance imaging in patients with cardiovascular devices: an American Heart Association scientific statementfrom the Committee on Diagnostic and Interventional Cardiac Catheterization, Council on Clinical Cardiology, and the Council on Cardiovascular Radiologyand Intervention: endorsed by theAmerican College of Cardiology Foundation, the North American Society for Cardiac Imaging, and the Society for Cardiovascular Magnetic Resonance. Circulation. 2007;116(24):2878-91.

19. Brignole M, Auricchio A, Baron-Esquivias G, Bordachar $P$, Boriani G, Breithardt OA, et al. 2013 ESC guidelines on cardiac pacing and cardiac resynchronization therapy: the task force on cardiac pacing and resynchronization therapy of the European Society of Cardiology (ESC). Developed in collaboration with the European Heart Rhythm Association (EHRA). Europace. 2013;15(8):1070-118.

20. Deshpande S, Kella D, Padmanabhan D. MRI in patients with cardiac implantable electronic devices: a comprehensive review. Pacing Clin Electrophysiol. 2021;44(2):360-72.

21. Muthalaly RG, Nerlekar N, Ge Y, Kwong RY, Nasis A. MRI in patients with cardiac implantable electronic devices. Radiology. 2018;289(2):281-92.

22. Poh PG, LiewC, Yeo C, Chong LR, Tan A, Poh A. Cardiovascular implantable electronic devices: a review of the dangers and difficulties in MR scanning and attempts to improve safety. Insights Imaging. 2017;8(4):405-18.

23. Luechinger R, Duru F, Scheidegger MB, Boesiger P, Candinas R. Force and torque effects of a 1.5-Tesla MRIscanner on cardiac pacemakers and ICDs. Pacing Clin Electrophysiol. 2001;24(2):199-205.

24. Luechinger R, Duru F, Zeijlemaker VA, Scheidegger MB, Boesiger P, Candinas R. Pacemaker reed switch behavior in $0.5,1.5$, and 3.0 Tesla magnetic resonance imaging units: Are reed switches always closed in strong magnetic fields? Pacing Clin Electrophysiol. 2002;25(10):1419-23.
25. Tandri H, Zviman MM, Wedan SR, Lloyd T, Berger RD, Halperin H. Determinants of gradient field-induced current in a pacemaker lead system in a magnetic resonance imaging environment. Heart Rhythm. 2008;5(3):462-8.

26. Luechinger R, Zeijlemaker VA, Pedersen EM, Mortensen P, Falk E, Duru F, et al. In vivo heating of pacemaker leads during magnetic resonance imaging. Eur Heart J. 2005;26(4):376-83. discussion 325-327.

27. van der Graaf AW, Bhagirath P, Götte MJ. MRI and cardiac implantable electronic devices; current status and required safety conditions. Neth HeartJ.2014;22(6):269-76.

28. Vahlhaus C, Sommer T, Lewalter T, Schimpf R, Schumacher $B$, Jung $W$, et al. Interference with cardiac pacemakers by magnetic resonance imaging: Are there irreversible changes at 0.5 Tesla? Pacing Clin Electrophysiol. 2001;24(4 Pt 1):489-95.

29. Higgins JV, Sheldon SH, Watson RE Jr, Dalzell C, Acker N, Cha YM, et al. "Power-on resets" in cardiac implantable electronic devices during magnetic resonance imaging. Heart Rhythm. 2015;12(3):540-4.

30. Hilbert S, Jahnke C, Loebe S, Oebel S, Weber A, Spampinato $R$, et al. Cardiovascular magnetic resonance imaging in patients with cardiac implantable electronic devices: a device-dependent imaging strategy for improved image quality. Eur Heart J Cardiovasc Imaging. 2018;19(9):1051-61.

31. International Organization for Standardization. Active implantable medical devices-four-pole connector system for implantable cardiac rhythm management devices-dimensional and test requirements (ISO Standard No. 27186:2020). 2020. https://www.iso.org/standard/ 72920.html. Accessed 4 Mar 2021.

32. Shah AD, Patel AU, Knezevic A, Hoskins MH, Hirsh DS, Merchant FM, et al. Clinical performance of magnetic resonanceimaging conditionalandnonconditional cardiac implantable electronic devices. Pacing Clin Electrophysiol. 2017;40(5):467-75.

33. Han D, Kang SH, Cho Y, Oh IY. Experiences of magnetic resonance imaging scanning in patients with pacemakers or implantable cardioverter-defibrillators. Korean J Intern Med. 2019;34(1):99-107.

34. Seewöster T, Löbe S, Hilbert S, Bollmann A, Sommer P, Lindemann F, et al. Cardiovascular magnetic resonance imaging in patients with cardiac implantable electronic devices: best practiceand real-world experience. Europace. 2019;21(8):1220-8.

35. Maass AH, Hemels MEW, Allaart CP. Magnetic resonance imaging in patients with cardiac implantable electronic devices. Neth Heart J.2018;26(12):584-90.

36. Do DH, Eyvazian V, Bayoneta AJ, Hu P, Finn JP, Bradfield JS, et al. Cardiac magnetic resonance imaging using wideband sequences in patients with nonconditional cardiac implanted electronic devices. Heart Rhythm. 2018;15(2):218-25.

37. Junttila MJ, Fishman JE, Lopera GA, Pattany PM, Velazquez DL, Williams AR, et al. Safety of serial MRI in patients with implantable cardioverter defibrillators. Heart. 2011;97(22):1852-6.

Publisher's Note Springer Nature remains neutral with regard to jurisdictional claims in published maps and institutional affiliations. 